Indonesian Journal of Physics

Vol 19 No. 4, October 2008

\title{
Some Gauge Related Aspects in Gauge Theory
}

\author{
Triyanta \\ ${ }^{1)}$ Theoretical High Energy Physics and Instrumentation (THEPI) Research Division \\ Faculty of Mathematics and Natural Sciences, \\ Institut Teknologi Bandung \\ ${ }^{2)}$ Indonesia Center for Theoretical and Mathematical Physics (ICTMP) \\ Faculty of Mathematics and Natural Sciences, \\ Institut Teknologi Bandung \\ e-mail: triyanta@fi.itb.ac.id
}

\begin{abstract}
Gauge freedom is one important property of gauge theory. It enables one to choose gauge fields that fulfill a certain condition for conveniences. Thus, gauge freedom leads more fruitful and interesting development of gauge theory. In this paper we will discuss some gauge condition related aspects where the generalized gauge is taken into consideration. More attention will be given to the FS gauge.
\end{abstract}

Keywords: Gauge theory, Gauge choice, Fock-Schwinger, Christoffel symbol, Propagator, Gauge symmetry

\section{Introduction}

Gauge theory imposes that a Lagrangian of matter fields $\psi_{l}(x)$ is invariant under the set of local infinitesimal phase transformations ${ }^{1)}$

$$
\delta \psi_{l}(x)=-i \varepsilon^{a}(x)\left(t^{a}\right)_{l}^{m} \psi_{m}(x) .
$$

In the above, summation over repeated indices is understood. The infinitesimal real parameters $\varepsilon^{a}$ depend on the space-time coordinates and $\left\{t^{a}\right\}$ is a set of linearly independent constant matrices fulfilling the Lie algebra

$$
\left[t^{a}, t^{b}\right]=i C^{a b} t^{c}
$$

The Jacobi identities for $t^{\mathrm{a}}$ restrict the structure constants $C^{a b}{ }_{c}=-C^{b a}{ }_{c}$ to satisfy the relations

$$
C^{a b}{ }_{e} C^{e c}{ }_{d}+C^{c a}{ }_{e} C^{e b}{ }_{d}+C^{b c}{ }_{e} C^{e a}{ }_{d}=0 .
$$

The indices $l, m$ have the values $1,2,3, \ldots, n$ where $n$ is the size of the multiplet $\psi_{l}(x)$, the $n$-plet, while the indices $a, b$, and $c$ have the values $1,2, \ldots$ up to the number of transformation generators $t^{a}$. The invariant Lagrangian should have the form $\mathscr{L}=\mathscr{L}\left(\psi, D_{\mu} \psi\right)$ where the covariant derivative $D_{\mu}$ is defined through

$$
D_{\mu} \psi_{l}(x)=\partial_{\mu} \psi_{l}(x)-i A_{\mu}^{b}(x)\left(t^{b}\right)_{l}^{m} \psi_{m}(x)
$$

with the vector fields $A_{\mu}$, called the gauge field, have to change according to

$$
\begin{aligned}
& \delta A_{\mu}^{b}(x)=-\partial_{\mu} \varepsilon^{b}(x)+C^{b}{ }_{c a} \varepsilon^{a}(x) A_{\mu}^{c}(x) \\
& =-D_{\mu}^{a b} \varepsilon^{b}(x), \quad D_{\mu}^{a b}=\delta^{b a} \partial_{\mu}-C^{b}{ }_{c a} A_{\mu}^{c}
\end{aligned}
$$

when the matter fields transform according to (1). The parameter $\varepsilon^{a}$ are called the gauge functions. The transformations (1) together with (5) are called the infinitesimal gauge transformations of the second kind. That of the first kind is the global gauge transformation characterized by the independent of $\varepsilon^{a}$ with respect to space and time coordinates. In this case the Lagrangian of free matter fields is already phase invariant, thus no gauge fields are necessary for the invariance.

It turns out that the local gauge invariant Lagrangian contains both matter fields and gauge fields. The appearance of the gauge fields means that the matter fields are no longer free. Thus, a local gauge invariant Lagrangian describes a system of interacting fields. For the case of singlet, the indices $l$ and $m$ only have one value, say 1 , thus can be discarded, and the matrices $t^{a}$ become numbers. The covariant derivative reduces into $\left(A_{\mu}^{b} t^{b}=A_{\mu}\right)$ :

$$
D_{\mu} \psi(x)=\partial_{\mu} \psi(x)-i e A_{\mu}(x) \psi(x)
$$

or

$$
i D_{\mu} \psi(x)=\left(i \partial_{\mu}+e A_{\mu}(x)\right) \psi(x) .
$$

This expressions reminding us to the canonical momenta in classical electrodynamics: the interaction between a charge particle with the electromagnetic field $A_{\mu}$ can be described by replacing the linear momenta $p_{i}$ of free charge particles with their canonical momenta $P_{i}=p_{i}+e A_{i}$. Thus one can identify that the gauge field represents electromagnetic field when the corresponding matter fields are charged particles. Gauge theory extends this prescription to other types of fundamental interactions. The gauge theory for spinor fields describing, e.g., electrons is described by the Lagrangian (without external sources)

$$
\mathscr{L}=\bar{\psi}\left(i \gamma^{\mu} D_{\mu}-m\right) \psi=\bar{\psi}\left(i \gamma^{\mu} \partial_{\mu}-m+e \gamma^{\mu} A_{\mu}\right) \psi .
$$

This Lagrangian is obtained by replacing partial derivatives in the Lagrangian of free spinor fields by the covariant ones. For general gauge fields, the Lagrangian of spinor fields is also of the above form, but with covariant derivative (4).

The complete Lagrangian is given by inserting a term, say $\mathscr{L}_{A}$, describing the dynamics of the gauge fields. $\mathscr{L}_{A}$ should be gauge invariance, otherwise the 
complete Lagrangian (without external sources) is no longer gauge invariant. Requiring this invariance, the Lagrangian $\mathscr{L}_{A}$ should have the form

$$
\mathscr{L}_{A}=-\frac{1}{4} F_{\mu v}^{a} F^{a \mu v}
$$

where the field strengths $F^{a \mu \nu}$ are defined as

$$
F_{\mu v}^{a}=\partial_{\mu} A_{v}^{a}-\partial_{v} A_{\mu}^{a}+g C_{b c}^{a} A_{\mu}^{b} A_{v}^{c} .
$$

The above field strengths reduce to the Maxwell field strengths for the Abelian (commutative) case, $C^{a b}{ }_{c},=0$ :

$$
F_{\mu v}=\partial_{\mu} A_{v}-\partial_{v} A_{\mu} .
$$

Unlike to the more general of field strengths (the field strength in the non-Abelian case) the Maxwell field strengths are gauge invariant. Since the Maxwell field strengths are nothing but the electric and magnetic fields the gauge invariance of the field strengths means that infinite numbers of sets of scalar and vector potentials $A^{\mu}(\Phi, \vec{A})$, related to one and another through gauge transformations, give the same electric and magnetic fields. Thus we have a freedom to choose the set of potentials. The set of potentials chosen is a set fulfilling a certain condition or equation. This condition is called the gauge condition, the gauge choice, or simply the gauge. The known gauge choices are, among others, the Lorenz gauge $\partial_{\mu} A^{\mu}=0$, the Coulomb gauge $\nabla \cdot \vec{A}=0$, the axial gauge $n_{\mu} A^{\mu}=0$, and the Fock-Schwinger (FS) gauge $x_{\mu} A^{\mu}=0$.

Although, the field strengths of the nonAbelian gauge are not gauge invariant there are also some degrees of freedom in non-Abelian case. Such degrees of freedom lead to divergence of the generating functional of the gauge fields. Fortunately, gauge fixing enables us to avoid this divergence.

\section{Explicit Potentials in Some Gauge Choices}

Different choice of gauge leads to different potential equations. For example, consider the inhomogeneous Maxwell equations in term of potentials:

$$
\begin{aligned}
\nabla^{2} \Phi & +\frac{1}{c} \frac{\partial}{\partial t}(\nabla \cdot \vec{A})=-4 \pi \rho, \\
\nabla^{2} \vec{A} & -\frac{1}{c^{2}} \frac{\partial^{2} \vec{A}}{\partial t^{2}} \\
& -\nabla\left((\nabla \cdot \vec{A})+\frac{1}{c} \frac{\partial \Phi}{\partial t}\right)=-\frac{4 \pi}{c} \vec{J} .
\end{aligned}
$$

Taking the Lorenz gauge

$$
\partial_{\mu} A^{\mu}=\nabla \cdot \vec{A}+\frac{1}{c} \frac{\partial \Phi}{\partial t}=0
$$

we have the inhomogeneous wave equations for both $\Phi$ and $\vec{A}$

$$
\nabla^{2} \Phi_{L}-\frac{1}{c^{2}} \frac{\partial^{2}}{\partial t^{2}} \Phi_{L}=-4 \pi \rho,
$$

$$
\nabla^{2} \vec{A}_{L}-\frac{1}{c^{2}} \frac{\partial^{2} \vec{A}_{L}}{\partial t^{2}}=-\frac{4 \pi}{c} \vec{J}
$$

with the solutions ${ }^{2)}$

$$
\begin{aligned}
\Phi_{L}(\vec{r}, t) & =\int d^{3} r^{\prime} \frac{\rho\left(\vec{r}^{\prime}, t^{\prime}\right)}{\left|\vec{r}-\vec{r}^{\prime}\right|}, \\
\vec{A}_{L}(\vec{r}, t) & =\frac{1}{c} \int d^{3} r^{\prime} \frac{\vec{J}\left(\vec{r}^{\prime}, t^{\prime}\right)}{\left|\vec{r}-\vec{r}^{\prime}\right|}, \\
t^{\prime} & =t-\frac{\left|\vec{r}-\vec{r}^{\prime}\right|}{c} .
\end{aligned}
$$

When we take the Coulomb gauge $\nabla \cdot \vec{A}=0$, instead of the Lorenz gauge, the equations for potentials read

$$
\begin{aligned}
& \nabla^{2} \Phi_{C}=-4 \pi \rho, \\
& \nabla^{2} \vec{A}_{C}-\frac{1}{c^{2}} \frac{\partial^{2} \vec{A}_{C}}{\partial t^{2}}=-\frac{4 \pi}{c} \vec{J}_{t}, \\
& \vec{J}_{t}=\vec{J}-\frac{1}{4 \pi} \nabla \frac{\partial \Phi_{C}}{\partial t} .
\end{aligned}
$$

The solutions are

$$
\begin{aligned}
\Phi_{C}(\vec{r}, t) & =\int d^{3} r^{\prime} \frac{\rho\left(\vec{r}^{\prime}, t\right)}{\left|\vec{r}-\vec{r}^{\prime}\right|}, \\
\vec{A}_{C}(\vec{r}, t) & =\frac{1}{c} \int d^{3} r^{\prime} \frac{\vec{J}_{t}\left(\vec{r}^{\prime}, t^{\prime}\right)}{\left|\vec{r}-\vec{r}^{\prime}\right|}, \\
t^{\prime} & =t-\frac{\left|\vec{r}-\vec{r}^{\prime}\right|}{c} .
\end{aligned}
$$

The current $\vec{J}_{t}$ is called the transverse current as it has a zero divergence:

$$
\begin{aligned}
\nabla \cdot \vec{J}_{t} & =\nabla \cdot \vec{J}-\frac{1}{4 \pi} \nabla^{2} \frac{\partial \Phi_{C}}{\partial t} \\
& =\nabla \cdot \vec{J}+\frac{\partial \rho}{\partial t}=0 .
\end{aligned}
$$

Thus we have two different sets of potentials $\left(\Phi_{C}, \vec{A}_{C}\right)$ and $\left(\Phi_{L}, \vec{A}_{L}\right)$ but with the same electric and magnetic fields. Both sets of potensials can be related to each other through the gauge function $\varepsilon$ according to the equation (5) with $C^{a b}{ }_{c}=0$ :

$$
A_{\mu}^{L}(x)=A_{\mu}^{C}(x)+\partial_{\mu} \varepsilon(x) .
$$

From this equation we obtain

$$
\frac{1}{c} \frac{\partial \varepsilon}{\partial t}=\Phi_{L}-\Phi_{C}=\int d^{3} r^{\prime} \frac{\rho\left(\vec{r}^{\prime}, t^{\prime}\right)-\rho\left(\vec{r}^{\prime}, t\right)}{\left|\vec{r}^{\prime}-\vec{r}\right|}
$$

giving

$$
\begin{aligned}
& \varepsilon(\vec{r}, t)=c \int d^{3} r^{\prime} \frac{1}{\left|\vec{r}^{\prime}-\vec{r}\right|} \\
& {\left[\begin{array}{l}
\int_{t_{0}}^{t-R / c} d t^{\prime} \rho\left(\vec{r}^{\prime}, t^{\prime}\right)-\int_{t_{0}}^{t} d t^{\prime} \rho\left(\vec{r}^{\prime}, t^{\prime}\right) \\
\quad+\varepsilon_{0}(\vec{r}), \quad R=\left|\vec{r}^{\prime}-\vec{r}\right|
\end{array}\right.}
\end{aligned}
$$

or 


$$
\begin{aligned}
\varepsilon(\vec{r}, t) & =c \int d^{3} r^{\prime} \frac{1}{\left|\vec{r}^{\prime}-\vec{r}\right|} \int_{t}^{t-R / c} d t^{\prime} \rho\left(\vec{r}^{\prime}, t^{\prime}\right)+\varepsilon_{0}(\vec{r}) \\
& =c \int d^{3} r^{\prime} \frac{1}{\left|\vec{r}^{\prime}-\vec{r}\right|} \int_{0}^{R / c} d \tau \rho\left(\vec{r}^{\prime}, t-\tau\right)+\varepsilon_{0}(\vec{r}) \\
& =c \int d^{3} r^{\prime} \frac{1}{\left|\vec{r}^{\prime}-\vec{r}\right|} \int_{0}^{1} d \lambda \rho\left(\vec{r}^{\prime}, t-\lambda R / c\right)+\varepsilon_{0}(\vec{r}) .
\end{aligned}
$$

We have learnt that choosing the Lorenz gauge or the Coulomb gauge simplifies the potential equations and this enables us to derive the solutions. However not all gauge choices reduce the complexity of the potential equations leading to difficulties in obtaining the solutions directly through the potentials equations. Examples of such gauge choices are the axial gauge and the FS gauge. No expressions $n_{\mu} A^{\mu}$ and $x_{\mu} A^{\mu}$ in the potentials equations unable us to simplify the equations. The appearance of the socalled inversion formulae in the FS gauge, however, makes it possible to derive potentials (the FS potentials) through the inversion formulae, not through the above potentials equations. The formulae are of the form ${ }^{3,4)}$

$$
A_{F S}^{\mu}(x)=-\int_{0}^{1} d \lambda \lambda x_{v} F^{\mu v}(\lambda x) .
$$

They express the potentials in terms of field strengths. Such expressions cannot be found in most other gauges. Thus, the inversion formula is the characteristics of the FS gauge. From the formulae we obtain the FS scalar and vector potentials

$$
\begin{aligned}
& \Phi_{F S}(\vec{r}, t)=-\int_{0}^{1} d \lambda \lambda \vec{r} \cdot \vec{E}(\lambda \vec{r}, \lambda t) \\
& \vec{A}_{F S}(\vec{r}, t)=-\int_{0}^{1} d \lambda \lambda[c t \vec{E}(\lambda \vec{r}, \lambda t)+\vec{r} \times \vec{B}(\lambda \vec{r}, \lambda t)]
\end{aligned}
$$

Since the same electric and magnetic fields can be obtained from different set of potentials one can rewrite the electric and magnetic fields in the right hand side of the above equations in terms of a set of (known) potentials, e.g. the Lorenz potentials:

$$
\begin{aligned}
\Phi_{F S}(\vec{r}, t)= & \int_{0}^{1} d \lambda \lambda \vec{r} \cdot \vec{\Psi}, \\
\vec{A}_{F S}(\vec{r}, t)= & \int_{0}^{1} d \lambda \lambda\{c t \vec{\Psi}+ \\
& \left.-\vec{r} \times\left[\nabla^{\prime} \times \vec{A}_{L}\left(\vec{r}^{\prime}, t^{\prime}\right)\right]\right\} .
\end{aligned}
$$

In the above $\nabla^{\prime}=(1 / \lambda) \nabla, \vec{r}^{\prime}=\lambda \vec{r}, t^{\prime}=\lambda t$ and

$$
\vec{\Psi}=\nabla^{\prime} \Phi_{L}\left(\vec{r}^{\prime}, t^{\prime}\right)+\frac{1}{c} \frac{\partial \vec{A}_{L}\left(\vec{r}^{\prime}, t^{\prime}\right)}{\partial t^{\prime}} .
$$

Insertion of the explicit form of the Lorenz potentials into the above equations gives rise to the complete set of FS potentials. The complexities of the FS potentials are understood as due to the complexities of the inhomogeneous Maxwell equations in terms of FS potentials. The inversion formulae (23), or alternatively (24) and (25), have been considered for various applications.

Similar relationship between the Gauge field and the field strength with that between the Christoffel symbol and the Riemannian tensor in general relativity enables us to derive inversion formulae in general relativity ${ }^{5)}$ :

$$
\Gamma_{F S}^{\mu}(x)=\int_{0}^{1} d \lambda \lambda x_{v} R^{\mu v}(\lambda x) .
$$

In the above $\Gamma_{F S}^{\mu}$ and $R^{\mu v}$ are matrices with the FS Christoffel symbols and Riemannian tensors, respectively, as their elements. The FS Christoffel symbols are Christoffel symbols fulfilling the FS condition $x_{\mu} \Gamma_{F S}^{\mu}=0$. The above inversion formulae lead to the geodesic equations into

$$
\begin{aligned}
\ddot{x}^{k} & =\left[(1+x \cdot \partial)^{-1} x^{\sigma} R_{\alpha \beta \sigma}^{k}\right. \\
& \left.-\dot{x}^{k}(1+x \cdot \partial)^{-1} x^{\sigma} R_{\alpha \beta \sigma}^{0}\right] \dot{x}^{\alpha} \dot{x}^{\beta} .
\end{aligned}
$$

The right hand side of the above equation describes Newtonian force per unit of mass. For speeds much less than the speed of light the above equation can be approximated to

$$
\ddot{x}^{k} \approx \frac{c^{2}}{2} \partial^{k}(x \cdot \partial)^{-1}(x \cdot \partial-1) g_{00}
$$

giving the FS Newtonian gravitational potential ( $\Phi$ is the familiar Newtonian gravitational potential)

$$
\begin{array}{r}
\Phi_{F S}=\left[1-(x \cdot \partial)^{-1}\right] \Phi+\frac{c^{2}}{2}, \\
\Phi=-\frac{c^{2}}{2}\left(1-g_{00}\right) .
\end{array}
$$

\section{Ghost-free and Non-ghost-free Gauges}

As has been mentioned in the previous section, problem of divergence in the generating functional of the gauge field can be solved by fixing a gauge. In overcoming this problem Faddeev and Popov defined a gauge invariant functional $\Delta[A]$

$$
\Delta[A]=\left\{\int \mathscr{Q} \varepsilon \delta\left(\partial^{\mu} A_{\mu}^{(\varepsilon) a}\right)\right\}^{-1} .
$$

In the above $\varepsilon(x)$ is a gauge parameter and thus $A_{\mu}^{(\varepsilon) a}$ are gauge-transformed gauge fields. The Dirac delta function in the right hand side shows that the Lorenz gauge has been taken into account. The above expression leads to the identity

$$
\Delta[A]\left\{\int \mathscr{Q} \varepsilon \delta\left(\partial^{\mu} A_{\mu}^{(\varepsilon) a}\right)\right\}=1 .
$$

By inserting this identity into the generating functional for the gauge field, the gauge parameter $\varepsilon$ that is responsible for the divergence can be fully pushed into the integral measure $\mathscr{D} \varepsilon$. Accordingly, integration over $\varepsilon$ gives rise to a factor with an infinite 
value. This factor can be absorbed into the normalization factor of the generating functional, leaving the generating functional finite. Besides solving the divergence problem, this mechanism leads to two important points: (1) the Dirac delta function, through averaging under the Gaussian weight, is equivalent to adding a gauge fixing Lagrangian $\mathscr{L}_{g f}$ into the gauge field Lagrangian (9), (2) the functional $\Delta[A]$ transforms into a Lagrangian of so-called ghost fields, the Grassmannian fields fulfilling the massless Klein-Gordon equation. Note that the gauge fixing Lagrangian above has the form ( $\lambda$ is a parameter)

$$
\mathscr{L}_{\text {gf }}=-\frac{1}{2 \lambda}\left(\partial_{\mu} A^{a \mu}\right)^{2} .
$$

Note also that in the Abelian case the functional $\Delta[A]$ is just a number and thus can be inserted into normalization factor of the generating functional. Accordingly, ghost fields can be discarded in Abelian gauge theory.

Now, when we replace the partial derivative $\partial_{\mu}$ in the Dirac delta function with $f_{\mu}$, where $f_{\mu}$ can be a

$$
\begin{aligned}
M^{a b}(x, y) & =\frac{\delta f^{\mu} A_{\mu}^{(\varepsilon) a}(x)}{\delta \varepsilon^{b}(y)}=C^{a b}{ }_{c}{ }^{\mu}\left[A_{\mu}^{c}(x) \delta(x-y)\right]-\frac{1}{g} \delta^{a b} f^{\mu} \partial_{\mu} \delta(x-y) \\
& =\left\{\begin{array}{cr}
-\frac{1}{g} \delta^{a b} f^{\mu} \partial_{\mu} \delta(x-y) & \text { axial, FS gauge } \\
C^{a b}{ }_{c} A_{\mu}^{c}(x) \partial^{\mu} \delta(x-y)-\frac{1}{g} \delta^{a b} \partial^{\mu} \partial_{\mu} \delta(x-y) & \text { Lorenz gauge }
\end{array}\right.
\end{aligned}
$$

It turns out that $M^{a b}$ for the axial gauge and the FS gauge are independence of gauge fields. Accordingly, the functional integration in the right hand side of (34) results in a number and therefore the functional $\Delta[A]$ can be absorbed into the normalization factor of the corresponding generating functional; in other word, the ghost fields can be discarded from the theory. We say that the axial and the FS gauge are ghost-free gauges. The Lorenz gauge, on the other hand, is not a ghost-free gauge because the corresponding $M^{a b}$ is gauge field dependent. Studying the first line of the above equation one can group the set of gauge conditions of the form $f_{\mu} A^{\mu}=0$ into two: set of ghostfree gauges and set of non ghost-free gauges. The former is related to any $f_{\mu}$ that does not contain derivatives while $f_{\mu}$ in the later does contain derivatives. Please note that the ghost-free property of the Abelian theory is understood as due to $C^{a b}{ }_{c}=0$.

\section{Field Equations and Propagators}

Consider the Lagrangian of the Abelian gauge field, the Maxwell field, without fixing a gauge:

$$
\mathscr{L}_{A}=-\frac{1}{4} F_{\mu v} F^{\mu v}+A_{\mu} J^{\mu} .
$$

The Euler-Lagrange equations give the inhomogeneous Maxwell equations ( $g^{\mu v}$ is the metric in Minkowski space):

$$
\partial_{\mu} F^{\mu v}=-J^{v} \rightarrow\left(g^{\mu v} \partial^{\alpha} \partial_{\alpha}-\partial^{\mu} \partial^{v}\right) A_{\mu}=-J^{v} .
$$

constant vector $n_{\mu}$, the space-time coordinate $x_{\mu}$, or the partial derivative $\partial_{\mu}$, the functional $\Delta[A]$ becomes

$$
\Delta[A]=\left\{\int \mathscr{D} \varepsilon \delta\left(f^{\mu} A_{\mu}^{(\varepsilon) a}\right)\right\}^{-1} .
$$

This gives

$$
\begin{aligned}
& \Delta[A]=\int \mathscr{D}\left[\chi \chi^{*}\right] \\
& \exp \left\{-\int d x d y \chi^{*^{a}}(x) M^{a b}(x, y) \chi^{b}(y)\right\}
\end{aligned}
$$

while gauge fixing Lagrangian

$$
\mathscr{L}_{\text {gf }}=-\frac{1}{2 \lambda}\left(f_{\mu} A^{a \mu}\right)^{2}
$$

or equivalently

$$
\mathscr{L}_{\text {gf }}=C^{a} f_{\mu} A^{a \mu}+\frac{\lambda}{2}\left(C^{a}\right)^{2}
$$

when auxiliary fields $C^{a}$ are introduced. In the above $\chi$ and $\chi^{*}$ are ghost fields and ${ }^{4)}$
These equations are identical to the equations (12). Since the operator in the left hand side has no inverse we must modify it by adding a term in such a way that the modified operator is not singular. This modification is equivalent to adding a gauge fixing term in the Lagrangian. Inserting the general gauge fixing Lagrangian (35) we have

$$
\mathscr{L}_{A}=-\frac{1}{4} F_{\mu v} F^{\mu v}-\frac{1}{2 \lambda}\left(f_{\mu} A^{\mu}\right)^{2}+A_{\mu} J^{\mu}
$$

with the corresponding field equations

$$
J^{v}=-\left\{\begin{array}{cc}
{\left[g^{\mu v} \partial^{2}-\left(1-\frac{1}{\lambda}\right) \partial^{v} f^{\mu}\right] A_{\mu}} & \text { Lorenzgauge } \\
{\left[g^{\mu v} \partial^{2}-\partial^{\mu} \partial^{v}-\frac{1}{\lambda} f^{\mu} f^{v}\right] A_{\mu}} & \text { Axial,FSgauges }
\end{array}\right.
$$

The solutions are of the forms

$$
A_{\mu}(x)=-\int d y G_{\mu \nu}(x, y) J^{v}(y)+A_{\mu}^{(0)}(x) .
$$

In the above the terms $A_{\mu}^{(0)}(x)$ obey the homogeneous equations and $\partial^{2}=\partial^{\mu} \partial_{\mu}$. It turns out that the Green's functions or the free propagators are related to the inverse of the operators inside the [] brackets, say them as $G^{-1}{ }_{\mu \nu}$, in the (41): 


$$
G^{\mu v}(x, y)=\left\{\begin{array}{lr}
{\left[g^{\mu v} \partial^{\alpha} \partial_{\alpha}-\left(1-\frac{1}{\lambda}\right) \partial^{v} f^{\mu}\right]^{-1} \delta(x-y)} & \text { Lorenz gauge } \\
{\left[g^{\mu v} \partial^{\alpha} \partial_{\alpha}-\partial^{\mu} \partial^{v}-\frac{1}{\lambda} f^{\mu} f^{v}\right]^{-1} \delta(x-y)} & \text { Axial, FS gauges }
\end{array}\right.
$$

After replacing $f^{\mu}$ with the values in the corresponding gauges and using the identity we have $\mathrm{e}^{4)}$

$$
\begin{aligned}
& G^{-1}{ }_{\mu \nu}(x) G^{\nu \alpha}(x, y)=\delta_{\mu}^{\alpha} \delta(x-y) \\
& G^{\mu v}(x, y)=\left\{\begin{array}{cc}
\left(\partial^{2}\right)^{-1}\left[g^{\mu v}+(\lambda-1) \partial^{\mu} \partial^{v}\right] \delta(x-y) & \text { Lorenz gaug } \\
\left(\partial^{2}\right)^{-1}\left[\begin{array}{l}
g^{\mu v}-(\partial \cdot n)^{-1}\left(\partial^{\mu} n^{v}+n^{\mu} \partial^{v}\right)+ \\
(\partial \cdot n)^{-2}\left(n^{2}-\lambda \partial^{2}\right) \partial^{\mu} \partial^{v}
\end{array}\right] \delta(x-y) & \text { Axial gauge } \\
\left(\partial^{2}\right)^{-1}\left[\begin{array}{l}
g^{\mu v}-(\partial \cdot x-1)^{-1}\left(\partial^{\mu} x^{v}+x^{\mu} \partial^{v}\right)+ \\
(\partial \cdot x-1)^{-2} \partial^{\mu} x^{2} \partial^{v}+ \\
-\lambda(\partial \cdot x-1)^{-1}(\partial \cdot x+3)^{-1} \partial^{2} \partial^{\mu} \partial^{v}
\end{array}\right] \delta(x-y) & \text { FS gauge }
\end{array}\right.
\end{aligned}
$$

Note that in non-Abelian case the free propagators have the forms $G^{a b \mu v}=\delta^{a b} G^{\mu v}$ with $G^{\mu v}$ equals the above equation. In addition, if the gauge fixing Lagrangian (36) is considered, instead of (35), we have additional components $G^{\mu 4}$ where the index 4 is related to the auxiliary field. These components, however, do not contribute to scattering.

Finally one concludes that the propagators in the ghost-free gauge above are must more complicated than that in the Lorenz gauge. This leads to complexities in perturbation theory calculations. This is the drawback of the ghost-free gauges.

Some properties of the gauge propagators are, among others,: (1) they have $\{\mu \leftrightarrow \nu, \quad x \leftrightarrow y\}$ symmetrical properties, (2) they fulfill the condition $f_{\mu} G^{\mu v}=0$, and (3) Lorenz and axial gauge propagators are translation invariant, but not for the FS gauge propagator.

\section{Symmetries and Identities}

Consider the Lagrangian of matter fields and gauge fields

$$
\mathscr{L}_{0}=-\frac{1}{4} F_{\mu v}^{a} F^{a \mu v}+\bar{\psi}\left(i \gamma^{\mu} D_{\mu}-m\right) \psi
$$

The above Lagrangian describes a system of quarks and gluons and it also represents electrodynamics when we reduce to Abelian case. It is invariant under the infinitesimal local gauge transformations

$$
\begin{aligned}
& \psi(x) \rightarrow \psi^{\prime}(x)=U(x) \psi(x)=\left(1-i g t^{a} \varepsilon^{a}(x)\right) \psi(x), \\
& \bar{\psi}(x) \rightarrow \bar{\psi}^{\prime}(x)=\bar{\psi}(x) U^{*}(x)=\bar{\psi}\left(1+i g t^{a} \varepsilon^{a}(x)\right), \\
& A_{\mu}^{a}(x) \rightarrow A_{\mu}^{\prime a}(x)=A_{\mu}^{a}(x)-D_{\mu}^{a b}(x) \varepsilon^{b}(x) .
\end{aligned}
$$

(The first and third equations are identical to (1) and (5)). If we fix the gauge by adding a gauge fixing Lagrangian $\mathscr{L}_{\text {gf }}$ (36), however, the new Lagrangian ( $\mathscr{L}_{0}$ $+\mathscr{L}_{g f}$ ) has no gauge symmetry. This is because the gauge fixing Lagrangian is not invariant under the gauge transformation. If we also add to the Lagrangian, the Faddeev-Popov ghost field contribution of the form

$$
\mathscr{L}_{F P}=-\int d y \chi^{*^{a}}(x) M^{a b}(x, y) \chi^{b}(y)
$$

the more complete Lagrangian

$$
\mathscr{L}=\mathscr{L}_{0}+\mathscr{L}_{\text {gf }}+\mathscr{L}_{\text {FP }},
$$

is also not invariant under the gauge transformation. This means that $\delta \mathscr{S}_{0}$ and $\delta \mathscr{S}_{\text {gf }}$ do not cancel to one another. However, there are extended gauge transformations, namely the BRST transformations, that leave the whole Lagrangian $\mathscr{S}$ unchanged. These transformations are the gauge transformations (47) plus the transformations for the ghost fields and the auxiliary fields of the forms:

$$
\begin{array}{ccc}
\chi^{a}(x) & \rightarrow & \chi^{\prime a}(x)=\chi^{a}(x)-\frac{1}{g} \theta C^{a b c} \chi^{b}(x) \chi^{c}(x) \\
\chi^{*^{a}}(x) & \rightarrow & \chi^{* a}(x)=\chi^{*^{a}}(x)-\theta C^{a}(x) \\
C^{a}(x) & \rightarrow & C^{\prime a}(x)=C^{a}(x)-\frac{n}{\lambda} \theta f^{\mu} D_{\mu}^{a b} \chi^{b}(x)
\end{array}
$$


In the above equations $\theta$ is a Grassmann number defined through $\varepsilon=-\theta \chi$. Since $\varepsilon$ is a real number $(\theta \chi)^{+}=\theta^{+} \chi^{+}$. We introduce the number $n$ so that the analysis can be used for either form of $\mathscr{L}_{g f}$ (eq. (35) or (36)). $n=0$ when $C^{\mathrm{a}}$ are auxiliary fields and $n=1$ when $C^{a}=-\left(f_{\mu} A^{a \mu}\right) / \lambda$. Note that in Abelian case the BRST transformations reduce to the gauge transformations.

The invariant Lagrangian gives a consequence to the corresponding generating functional. In electrodynamics the generating functional is of the form

$$
\begin{aligned}
& Z[J, \bar{\eta}, \eta, K] \\
& =\int D[A \psi \bar{\psi} C] \Delta[A] \exp \left\{i \int d x\left[\mathscr{S}_{0}+\mathscr{L}_{g f}+\mathscr{L}_{s}\right]\right.
\end{aligned}
$$

with $\mathscr{L}_{0}$ equals (46) but without the internal index $a$, the functional $\Delta[A]$ is just a number, while $\mathscr{L}_{s}$, the external source term, is given by $\left(J_{\mu}^{a}, \eta, \bar{\eta}\right.$, and $K^{a}$ are external sources corresponding to the fields, respectively, $A^{a \mu}, \bar{\psi}, \psi$, and $C^{a}$ ):

$$
\mathscr{L}_{\mathrm{s}}=J_{\mu}^{a} A^{a \mu}+\bar{\psi} \eta+\bar{\eta} \psi+K^{a} C^{a} .
$$

(The index $a$ is also irrelevant in this case) Since $Z$ is invariant under any field transformations, $\delta \mathrm{Z}=0$, the gauge invariance of $\mathscr{L}_{0}$ leads to the Ward-Takahashi identity

$$
\pm i \partial_{\mu} f^{\mu} \frac{\delta \mathrm{Z}}{\delta K}+\partial_{\mu} J^{\mu} Z+e \eta \frac{\delta \mathrm{Z}}{\delta \eta}-e \bar{\eta} \frac{\delta \mathrm{Z}}{\delta \bar{\eta}}=0
$$

In term of effective action $\Gamma\left[A^{a \mu}, \bar{\psi}, \psi, C^{a}\right]$ the identity has the form

$$
\pm i \partial_{\mu} f^{\mu} C+i \partial_{\mu} \frac{\delta \Gamma}{\delta A_{\mu}}-e \bar{\psi} \frac{\delta \Gamma}{\delta \bar{\psi}}+e \psi \frac{\delta \Gamma}{\delta \psi}=0
$$

The $+(-)$ sign in the first term is associated with the Lorenz gauge (axial and FS gauges). It turns out that the first term is the only term that depends on the gauge choice. Since this term only depends on unphysical auxiliary field or only linear in gauge field (when $C$ is not auxiliary field) it does not contribute to $n$-point functions. Thus different gauge choices do not lead to different physical meaning. For example, the identity implies orthogonality of photon self-energy for all gauge choices.

In the case of non-Abelian gauge, both the functional $\Delta[A]$ and $\delta A$ depend on gauge fields and this gauge dependence leads to complexities of the socalled Slavnov-Taylor identity:

$$
\left[\begin{array}{l}
\frac{\delta}{i \delta K^{a}}+\int d y J^{b \mu} D_{\mu}^{b c}\left(\frac{\delta}{i \delta J}\right) M^{-1^{c a}} \\
\left(y, x ; \frac{\delta}{i \delta J}\right)+e \eta \frac{\delta}{\delta \eta}-e \bar{\eta} \frac{\delta}{\delta \bar{\eta}}
\end{array}\right] Z=0 .
$$

The complexities, due to the appearance of $M$, unable one to write in a simpler form like the WardTakahashi identity. This problem leads one to consider the BRST symmetry for the non-Abelian case.
Identities related to the BRST transformations can be obtained by inserting (34) into (51) giving

$$
\begin{aligned}
Z= & {\left.\left[J, \bar{\eta}, \eta, K, \xi^{*}, \xi\right]\right] } \\
= & \int D\left[A \psi \bar{\psi} C \chi \chi^{*}\right] \Delta[A] \\
& \exp \left\{i \int d x\left[\mathscr{L}_{0}+\mathscr{L}_{g f}+\mathscr{L}_{F P}+\mathscr{L}_{s}\right]\right.
\end{aligned}
$$

where $\mathscr{L}_{s}$ should contain ghost sources $\xi$ and $\xi^{*}$ :

$$
\begin{aligned}
& \mathscr{L}_{s}=J_{\mu}^{a} A^{a \mu}+\bar{\psi} \eta+\bar{\eta} \psi \\
& +K^{a} C^{a}+\chi^{*^{a}} \xi^{a}+\xi^{*^{a}} \chi^{a} .
\end{aligned}
$$

BSRT invariance of $\left(\mathscr{L}_{0}+\mathscr{L}_{g f}+\mathscr{S}_{F P}\right)$ gives rise to the BRST identity

$$
\begin{aligned}
& \int d x\left[\frac{\delta Z}{\delta K^{a}} \xi^{a}+J^{a \mu} \frac{\delta Z}{\delta u^{a \mu}}\right. \\
& \left.-\xi *^{a} \frac{\delta Z}{\delta v^{a}}+\frac{\delta Z}{\delta \omega} \eta-\bar{\eta} \frac{\delta Z}{\delta \omega}\right]=0 .
\end{aligned}
$$

In term of $\Gamma\left[A^{a \mu}, \bar{\psi}, \psi, C^{a}, \chi^{*}, \chi, \omega, \bar{\omega}\right]$, the effective action, the identity has the form

$$
\begin{aligned}
& \int d x\left[\frac{\delta \Gamma}{\delta u^{a \mu}}\left(\frac{\delta \Gamma}{\delta A_{\mu}^{a}} \pm f^{\mu} C^{a}\right)\right. \\
& \left.+\frac{\delta \Gamma}{\delta \chi^{a}} \frac{\delta \Gamma}{\delta v^{a}}+\frac{\delta \Gamma}{\delta \omega} \frac{\delta \Gamma}{\delta \bar{\psi}}+\frac{\delta \Gamma}{\delta \psi} \frac{\delta \Gamma}{\delta \bar{\omega}}\right]=0 .
\end{aligned}
$$

In obtaining the above identity we should introduce new anti-commuting source $u^{a \mu}$ and commuting sources $v^{a}, \omega$, and $\varpi$ of the composite fields, respectively, $D^{a b} \chi^{b},-g C^{a b c} \chi^{b} \chi^{c} / 2,-i g \bar{\psi} \chi^{a} t^{a}$, and $\operatorname{ig} \chi^{a} t^{a} \psi$. Similar to the previous identities, gauge choice dependence of the BRST identity is given by $f^{\prime \prime} C$ term that does not contribute to physical processes. Note that the BRST identity reduces to the Ward-Takahashi identity when we shift to the Abelian case.

In order to look at the dependence of the effective action with respect to the gauge parameter $\lambda$ one introduces the extended BRST transformations, i.e. the BRST transformations plus additional transformations of the form ${ }^{6}$

$$
\delta \lambda=\theta \gamma, \quad \delta \gamma=0 .
$$

In the above, $\gamma$ is a Grassmann variable, $\gamma^{2}=0$, defined through adding to the Lagrangian a term of the form $\gamma \chi^{*} C / 2$ that does not contribute to the dynamics of the physical system (The appearance of ghost fields in the additional transformation of course leads to changes of the dynamics of the ghost fields.):

$$
\mathscr{L}=\mathscr{L}_{0}+\mathscr{L}_{\text {gf }}+\mathscr{L}_{\mathrm{FP}}+\frac{1}{2} \gamma \chi^{* a} C^{a} .
$$

The new transformation shows that the gauge parameter $\lambda$ is considered as a variable. Taking into account to the extended BRST transformations one ends up with the so-called Nielsen identity ${ }^{7)}$ : 


$$
\begin{aligned}
& \int d x\left[\frac{\delta \Gamma}{\delta u^{a \mu}}\left(\frac{\delta \Gamma}{\delta A_{\mu}^{a}} \pm f^{\mu} C^{a}\right)\right. \\
& +\frac{\delta \Gamma}{\delta \chi^{a}} \frac{\delta \Gamma}{\delta v^{a}}+\frac{\delta \Gamma}{\delta \omega} \frac{\delta \Gamma}{\delta \bar{\psi}}+\frac{\delta \Gamma}{\delta \psi} \frac{\delta \Gamma}{\delta \omega} \\
& \left.-\frac{1}{2} \gamma\left(C^{a}\right)^{2}+\gamma \frac{\delta \Gamma}{\delta \lambda}\right]=0 .
\end{aligned}
$$

It turns out that the difference between the BRST and the Nielsen identities is given by the last two terms of the above equation. The last term describes how the effective action depends on the gauge parameter.

\section{Conclusions}

We have shown that freedom of choosing a gauge leads to the development of gauge theory more interesting. In electromagnetism we have, for the same electric and magnetic field, an infinite number of potential configurations. Gauge function that relates the Lorenz and the Coulomb gauge is explicitly given. Through the inversion formulae such a gauge function that relates the Lorenz and the FS gauge may also be obtained. The inversion formula is peculiar for the FS gauge and it interests some physicists to work with. Similarity between the $A_{\mu}-F_{\mu v}$ relationship in gauge theory and $\Gamma_{\mu}-R_{\mu v}$ relationship in general relativity leads to an inversion formula version of general relativity. This enables one to derive the FS Newtonian gravitational potential.

Various gauge choices can be grouped according to some certain properties. Here we have ghost-free gauges and non ghost-free gauges. Ghostfree gauges are gauge fixing of the form $f \cdot A=0$ with $f$ does not contain differential operators. In ghost-free gauges, no ghost lines are necessary in Feynman rules, thus reducing the number of Feynman diagrams.
However, we must pay the reduction of the diagrams by complexity of the propagators, giving difficulties in perturbation calculations. FS gauge proves these difficulties ${ }^{4}$.

Field transformation invariant of some part of the Lagrangian of the corresponding physical system leads to some identities, the Ward-Takahashi, Slavnov-Taylor, BRST, and the Nielsen identities. The identities are of the form of functional differentiation of the effective action with respect to the fields. The identities vary with gauge choices, but give the same physical consequences.

\section{References}

1. See, for example, Weinberg, S, The Quantum Theory of Fields, Cambridge University Press, 1998

2. J. D. Jackson, From Lorenz to Coulomb and other explicit gauge transformations, LBLN-50079, 2002.

3. W. Kummer and J. Weiser, Quantization of Gauge Fields in the Fock-Schwinger gauge, Z. Phys. C31, 105, 1986.

4. R. Delbourgo and Triyanta, The Equivalence between the Feynman Gauge and the FockSchwinger Gauge, Int. J. Mod. Phys. A 7:23, 5833, 1992.

5. Triyanta, General Theory of Relativity Without Christoffel Symbols, Proc. of Int.Conf.on Differential Equation, Kluwer, 1997.

6. N.K. Nielsen, On the gauge dependence of spontaneous symmetry breaking in gauge theories Nucl. Phys. B101, 173, 1975.

7. Triyanta and Sutisna, Identitas Nielsen, KFI, 12:4, 1, 2001. 